\title{
Empiema bacteriano espontáneo como complicación de la cirrosis hepática: ¿cuándo sospecharlo y cómo tratarlo? Presentación de casos
}

\author{
Martín Elizondo Barceló, ${ }^{1,2}$ Jimena Prieto Amorín, ${ }^{1,2}$ Julio César Medina Presentado, ${ }^{1,2}$ \\ Solange Gerona Sangiovanni ${ }^{1}$ \\ ${ }^{1}$ Unidad Bi Institucional de Enfermedades Hepáticas Complejas (Hospital Militar, Hospital de Clinicas), Programa de Trasplante Hepático. \\ ${ }^{2}$ Cátedra de Enfermedades Infecciosas, Facultad de Medicina. \\ Montevideo, Uruguay
}

Acta Gastroenterol Latinoam 2021;51(1):100-105

Recibido: 22/07/2020 / Aceptado: 14/02/2021 / Publicado online: 22/03/2021 / https://doi.org/10.52787/wlbq8266

\begin{abstract}
Resumen
El empiema bacteriano espontáneo se define como la infección del hidrotórax hepático en los pacientes cirróticos. Es una causa rara de descompensación en la cirrosis, existiendo escasos reportes bibliográficos en la región, y su presencia marca un punto de inflexión en la evolución de la enfermedad con un mal pronóstico a corto plazo. Se presentan dos casos clínicos de pacientes con esta complicación que fueron derivados al Programa Nacional de Trasplante Hepático de Uruguay. Se realiza la revisión del tema jerarquizando aspectos de la presentación clínica, la fisiopatología y un enfoque terapéutico. Se hace una especial mención a la importancia del tratamiento antibiótico empírico basado en el sitio de adquisición de la infección, la epidemiología local y en la oportunidad de la resolución del hidrotórax hepático subyacente, dado el alto riesgo de complicaciones que pueden asociar los tratamientos invasivos.
\end{abstract}

Palabras claves. Cirrosis, empiema bacteriano espontáneo, hidrotórax hepático.

Correspondencia: Martín Elizondo Barceló

Correo electrónico: melizondo20@gmail.com

\section{Spontaneous Bacterial Empyema as a Complication of Hepatic Cirrhosis: When to Suspect and How to Treat. A Case Report}

\section{Summary}

Spontaneous bacterial empyema is defined as infection of the liver hydrothorax in the cirrhotic patients. It is a rare cause of decompensated cirrhosis, with few bibliographic reports in the region, and its presence marks a turning point of inflection in the evolution of the disease with a poor prognosis in the short term. Two clinical cases of patients with this complication are presented, which were referred to the $\mathrm{Na}$ tional Liver Transplant Program of Uruguay. The clinical features, a pathophysiology and a therapeutic approach of this pathology are reviewed. Special emphasis is placed on empirical antibiotic treatment based on the site of acquisition of the infection and local epidemiology, and in the opportunity for the resolution of the underlying hepatic hydrothorax, with a high risk of complications that may be associated with invasive treatments.

Keywords. Cirrhosis, spontaneous bacterial empyema, hepatic hydrothorax.
Abreviaturas
EBE: Empiema bacteriano espontáneo.
HH: Hidrotórax hepático.
HTP: Hipertensión portal.
PBE: Peritonitis bacteriana espontánea.
EPS: Encefalopatía portosistémica. 
TP: Tiempo de protrombina.

MELD: Model for End-Stage Liver Disease.

C-P: Child-Pugh.

TH: Trasplante hepático.

PA: Presión arterial.

TC: Tomografia computada.

DP: Derrame pleural.

PMN: Polimorfonucleares.

CID: Coagulación intravascular diseminada.

MDR: Microorganismo multidrogo resistente.

XDR: Microorganismo extremadamente resistente.

AoCLF: Acute on Cronic Liver Failure.

SAE: Sindrome ascitico edematoso.

ECAs: Ensayos clínicos aleatorizados.

EPS: Encefalopatía portosistémica.

Q-SOFA: Quick Sepsis Related Organ Failure Assessment.

LP: Líquido pleural.

rpm: Respiraciones por minuto.

iv: Intra venoso.

v/o: Vía oral.

TIPS: Transjugular intrahepatic portosystemic shunt.

\section{Introducción}

El empiema bacteriano espontáneo (EBE) es la infección de un hidrotórax hepático $(\mathrm{HH})$ preexistente en un paciente cirrótico, e integra el grupo de infecciones espontáneas vinculadas a la hipertensión portal (HTP). ${ }^{1-3}$

Es una complicación con baja frecuencia, siendo menor al 1\% de las infecciones en cirróticos, con publicaciones de casos aislados en la región. ${ }^{3-5}$

Se reportan dos pacientes en los que se realizó un diagnóstico del EBE, cuya importancia radica en el análisis de las dificultades diagnósticas y terapéuticas, destacando las complicaciones vinculadas a los tratamientos invasivos que presentaron. Es una patología en la que es fundamental mantener un alto índice de sospecha en su búsqueda ante todo paciente con un $\mathrm{HH}$ y descompensación de la cirrosis, de manera de realizar un manejo adecuado y mejorar la sobrevida.

\section{Caso clínico $n^{\circ} 1$}

Un varón de 38 años tiene una cirrosis por alcohol asociada a una sobrecarga férrica por probable hemocromatosis (ferritina: $1,934 \mathrm{ng} / \mathrm{mL}$ e índice de saturación de transferrina: 97\%, con gen HFE:C282Y homocigota normal y H63D heterocigota). La HTP es clínicamente significativa con una gastropatía sin sangrados digestivos. Presenta una esplenomegalia con hiperesplenismo y una ascitis complicada con peritonitis bacteriana espontánea (PBE) comunitaria previa. Bajo tratamiento con espironolactona $200 \mathrm{mg} /$ día y furosemida $80 \mathrm{mg} /$ día. Encefalopatía portosistémica (EPS) grado 1. Alteración de la función de síntesis: tiempo de protrombina (TP) 26\% y albúmina 2,87 g/L. MELD (Model for End-Stage Liver Disease) Na: 26, Child-Pugh (C-P): C12, estadio de D' Amico: 4. En lista de espera para trasplante hepático (TH). Consultó por una fiebre de 10 días de evolución en ausencia de síntomas que orienten a un foco infeccioso.

$\mathrm{Al}$ ingreso estaba lúcido, eupneico, con una presión arterial (PA) de 100/60 mmHg; Q-SOFA = 0; ictericia conjuntival y auscultación cardiovascular normal. A nivel pleuropulmonar: disminución de vibraciones vocales, matidez y ventilación abolida a nivel de tercio inferior de hemitórax derecho.

Analítica sanguínea al ingreso: ver Tabla 1. MELD Na: 26. Se le realizó una radiografía de tórax (Figura 1): hay una opacidad en la base del hemitórax derecho. Luego, una tomografía computada (TC) de tórax (Figura 2): se observó un derrame pleural (DP) derecho moderado con atelectasia del lóbulo inferior derecho y parcial del lóbulo medio. La toracocentesis diagnóstica y terapéutica constata 960 polimorfonucleares $(\mathrm{PMN}) / \mu \mathrm{L}$ (Tabla 2).

Tabla 1. Analítica sanguinea al momento del diagnóstico del EBE

Caso 1 Caso 2 Valores de referencia

\begin{tabular}{|c|c|c|c|}
\hline BT/BD (Ul/mL) & $15,7 / 5,24$ & $0,77 / 0,52$ & $0,30-1,20 / 0,10-0,30$ \\
\hline AST/ALT (UI/mL) & $65 / 30$ & $62 / 31$ & $13-40 / 7-40$ \\
\hline FAL/GGT (UI/mL) & $111 / 27$ & $120 / 60$ & $40-116 / 5-73$ \\
\hline Albúmina (g/dL) & 3,20 & 3,04 & $3,50-5,20$ \\
\hline Creatininemia (mg/dL) & -) 0,76 & 2,52 & $0,70-1,30$ \\
\hline $\mathrm{Na}(\mathrm{mEq} / \mathrm{L})$ & 136 & 130 & $132-146$ \\
\hline TP (\%) / INR & $40 / 1,96$ & $39,2 / 1,79$ & $70-120$ \\
\hline $\mathrm{Hb}(\mathrm{g} / \mathrm{dL}) / \mathrm{Hto}(\%)$ & $7,3 / 21$ & $9 / 26$ & $13,4-16,6$ \\
\hline Plaquetas $\left(10^{3} / \mu \mathrm{L}\right)$ & 87 & 102 & $140-400$ \\
\hline $\mathrm{GB}\left(10^{3} / \mu \mathrm{L}\right)$ & 6,2 & 4,8 & $3,5-10,0$ \\
\hline PCR (mg/L) & 41,1 & 196 & $0,0-5,0$ \\
\hline
\end{tabular}

BT: bilirrubina total; BD: bilirrubina directa; AST: aspartato aminotransferasa; ALT: alanina aminotransferasa; TP: tiempo de protrombina; INR: international normalized ratio; $\mathrm{Hb}$ : hemoglobina; Hto: hematocrito; GB: glóbulos blancos; PCR: proteína $\mathrm{C}$ reactiva. 
Figura 1. Radiografía de tórax al momento del ingreso del paciente del caso clínico $N^{\circ} 1$ que evidencia una opacidad homogénea en vidrio esmerilado en la base del hemitórax derecho.

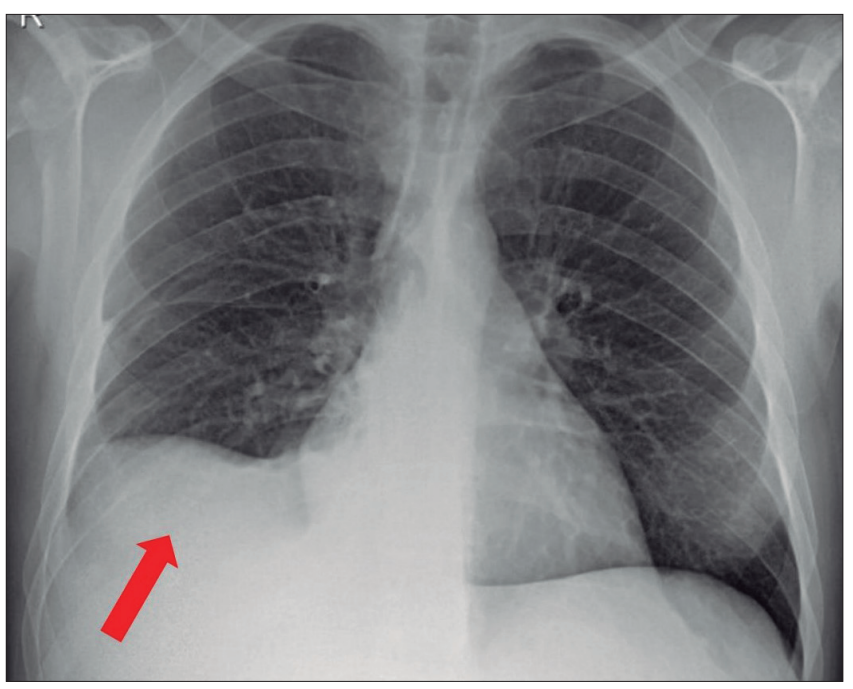

Figura 2. RTC de tórax (ventana de parénquima pulmonar) al momento del ingreso del caso clínico $N^{\circ} 1$ donde se observa un DP derecho.

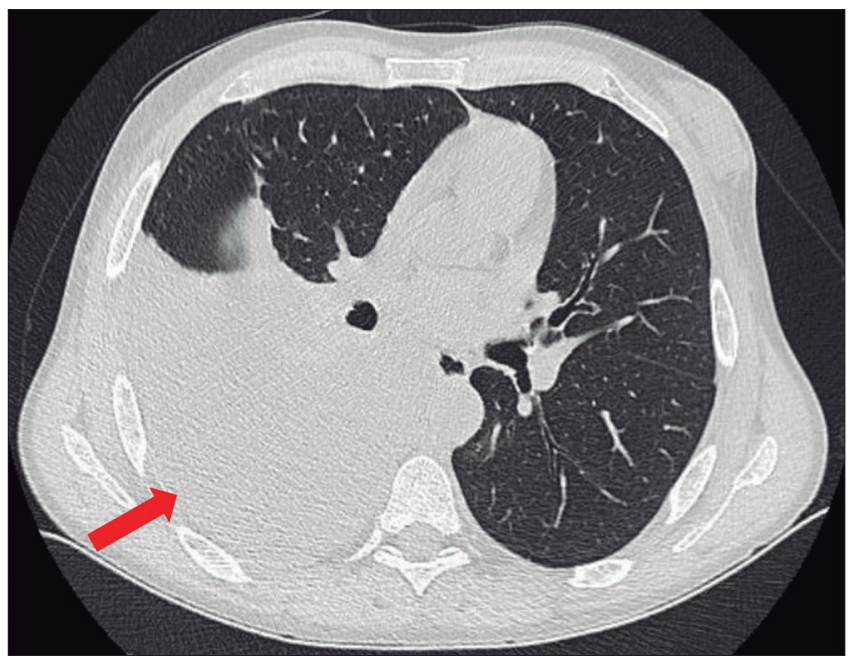

Tabla 2. Características del líquido pleural al momento del diagnóstico del EBE

\begin{tabular}{lcc}
\hline & Caso 1 & Caso 2 \\
\hline Aspecto macroscópico & Ligeramente turbio & Ligeramente turbio \\
PMN $(\mu \mathrm{L})$ & 960 & 3620 \\
Glucosa $(\mathrm{md} / \mathrm{dL})$ & 112 & 95 \\
Colesterol $(\mathrm{mg} / \mathrm{dL})$ & 50 & - \\
Proteínas totales $(\mathrm{g} / \mathrm{L})$ & 23 & 5 \\
LDH $(\mathrm{U} / \mathrm{L})$ & 138 & 141 \\
Desarrollo microbiológico & No & Sí \\
\hline
\end{tabular}

PMN: polimorfonucleares; LDH: lactato deshidrogenasa.
Con diagnóstico de un EBE, se inició un tratamiento antibiótico empírico con piperacilina-tazobactam asociada a albúmina. Se aumentó la dosis del tratamiento diurético sin la adecuada respuesta, que se suspendió por injuria renal aguda e hiponatremia. Dada la dificultad en el manejo con reproducción del DP en múltiples oportunidades se colocó un tubo de avenamiento pleural por el equipo de cirugía de tórax, realizándose una videopleuroscopía que mostró pleura edematosa sin sangrado. No desarrolló microorganismos bacterianos a nivel del líquido pleural ni de biopsia pleural.

En la evolución se agregó una coagulación intravascular diseminada (CID) con shock hipovolémico por el hemotórax, con un recuento de los PMN del LP elevado $\left(2700 / \mathrm{mm}^{3}\right)$, pero en el contexto de un líquido de características hemáticas, ingresó a terapia intensiva con una buena evolución inicial. Posteriormente presentó registros febriles. Una nueva TC de tórax evidenció un DP bilateral y áreas en vidrio deslustrado en el lóbulo superior derecho, compatible con una neumonía intrahospitalaria, no aislándose un microorganismo. Se asumió como una neumonía bacteriana probablemente por microorganismos multidrogo/extremadamente resistente (MDR/XDR) y se escaló un tratamiento antibiótico a colistín asociado a tigeciclina. Empeoró de forma clínica y analítica: evolucionó hacia una Acute on Cronic Liver Failure (AoCLF) con un posterior shock refractario por lo que falleció.

\section{Caso clínico $\mathbf{n}^{\circ} 2$}

Un varón de 57 años tenía una cirrosis hepática de etiología alcohólica con HTP clínicamente significativa, con várices esofágicas sin sangrado, un síndrome ascítico edematoso (SAE) y una EPS, sin infecciones previas. Se produce una caída de la función de síntesis. MELD Na: 14 y C-P: C10 en lista para un TH. Ingresó debido a una oclusión intestinal por una hernia umbilical con tratamiento quirúrgico sin incidentes, con buena evolución sin necesidad de colocar un drenaje en la cavidad abdominal. Concomitantemente se constató un hidrotórax derecho, que dada la presencia de una injuria renal (creatininemia 2,41 mg/dL), la que limitó el uso de diuréticos, se realizó la colocación de un tubo de avenamiento pleural, con un gasto de 9 litros/día de líquido seroso. No contamos con recuento de PMN en dicho contexto, aunque no presentó desarrollo en los cultivos. Dado el alto gasto del drenaje se requirió pleurodesis y decorticación, aislándose una Escherichia coli multisensible (resistente a trimpetropim-sulfametoxazol). Se indicó ciprofloxacina iv durante 10 días con 
una buena evolución, con alta en el domicilio luego de retirado el tubo de avenamiento pleural.

Reingresó a los 10 días por fiebre, sin tos, sin expectoración ni dolor torácico. En el examen físico estaba lúcido, frecuencia respiratoria (FR) de $24 \mathrm{rpm}$ y normotenso. La temperatura axilar era de $38^{\circ} \mathrm{C}$. Q-SOFA $=1$. Había una hipoventilación del hemitórax derecho y una ascitis moderada.

La analítica sanguínea se muestra en la Tabla 1. MELD Na: 26. En la TC de tórax se observó un DP derecho de distribución atípica, atelectasia total pasiva subyacente del lóbulo superior derecho y medio y subtotal del lóbulo inferior derecho. No se presentaron alteraciones del parénquima pulmonar. La toracocentesis tenía un recuento de 3,620 $\mathrm{PMN} / \mu \mathrm{L}$ y paracentesis que descartaba una PBE. El estudio citoquímico se puede ver en la Tabla 2. Se recolocó el tubo de tórax, se realizó una video-pleuroscopía y una nueva decorticación pleural derecha por una paquipleuritis con resección de pleura parietal. Con el diagnóstico del EBE en pleura intervenida, se inició la cefriaxona $2 \mathrm{~g}$ iv día y trimetoprima-sulfametoxazol $800 / 160 \mathrm{mg}$ v/o cada 12 horas asociados a albúmina. Bacteriológico de LP: Staphylococcus aureus sensible a la meticilina y Staphylococcus lugdunensis.

Evolucionó con mejoría clínica y analítica, retirándose el tubo de tórax, sin nuevo recuento de PMN. Se consolidó un tratamiento antibiótico con cefuroxima $\mathrm{v} / \mathrm{o}$ y trimetoprima-sulfametoxazol v/o. La TC de tórax de control fue sin colecciones. Se realizó el TH a los 9 meses con una excelente evolución.

\section{Discusión}

Las infecciones son una causa común de la descompensación de una cirrosis o precipitación de una AoCLF. ${ }^{1-6} \mathrm{El}$ $\mathrm{EBE}$ es una de las 3 infecciones espontáneas vinculadas a la HTP y se define como la infección del HH. ${ }^{1-3}$ El diagnóstico se sospecha en un paciente cirrótico con un DP y resultados clínicos de actividad infecciosa o descompensación. Se confirma mediante el recuento de PMN en LP o el desarrollo de microorganismos en su cultivo. ${ }^{1}$

Esta complicación tiene baja frecuencia. En una serie de aproximadamente 1300 infecciones en pacientes cirróticos solo el $0,9 \%$ la presentó, ${ }^{3}$ con reportes aislados en la región. ${ }^{4,5}$ La incidencia a los 4 años es del 2,4\% (81 en 3390) en todos los pacientes cirróticos y del 16\% si se presenta hidrotórax, ${ }^{7}$ aumentando la prevalencia en la enfermedad avanzada. ${ }^{8}$

Clínicamente se manifiesta con síntomas respiratorios (disnea o dolor torácico), con dolor abdominal o la presencia de síntomas sistémicos. ${ }^{9}$ Aunque no es infrecuente la presentación paucisintomática como describimos en el caso 1, donde el único elemento de actividad infecciosa fue la fiebre. Incluso, en la cirrosis avanzada, puede ocurrir la ausencia de sintomatología, siendo un hallazgo en el examen físico. El caso 2 es un ejemplo de lo importante que es la sospecha diagnóstica, sobre todo ante la descompensación sin causa aparente. ${ }^{9}$

La patogenia no está del todo aclarada y se plantea que el empiema como en la PBE es consecuencia de la infección del hidrotórax posterior a una bacteriemia espontánea. Sin embargo, otra hipótesis refiere que se debe al flujo de ascitis infectada en el contexto de una PBE. ${ }^{10}$ En este sentido se ha informado que la PBE se asocia al EBE en un $56 \%$ de los casos, ${ }^{11}$ aunque otra serie mostró que el $40 \%$ de los EBE no presentó la PBE previamente, siendo importante realizar un recuento de PMN en LP aún descartada la PBE. ${ }^{10}$

El diagnóstico se realiza con un recuento de PMN mayor a $500 / \mu \mathrm{L}$, como ocurrió en los pacientes presentados, o mayor a $250 / \mu \mathrm{L}$ con desarrollo de un microorganismo en el cultivo. ${ }^{1}$ Dichos cultivos son positivos solo en el 33\% de los pacientes por medios habituales, pudiendo aumentar a un $75 \%$ al inocularlo en botellas de hemocultivo al lado de la cama del paciente. ${ }^{12}$ Es de destacar que el análisis del LP mediante los criterios de Light no tiene utilidad. ${ }^{13}$ Para completar el diagnóstico debe descartarse una neumonía aguda comunitaria con los métodos imagenológicos, ya sea radiografía simple o TC de tórax. ${ }^{8,10}$ La ecografía torácica sirve para cuantificar la extensión del DP y, al ser utilizada como guía, reduce la tasa de complicaciones de la toracocentesis.? 14

El EBE y la PBE comparten bases fisiopatológicas por lo que los aislamientos microbiológicos son similares, siendo ambas infecciones monomicrobianas, donde las enterobacterias son los principales patógenos aislados, seguido de los cocos Gram positivos. ${ }^{7}$ Es importante diferenciar entre EBE de adquisición comunitaria y de adquisición hospitalaria. Dentro de los comunitarios, la bacteria $E$. coli es el microorganismo más frecuente y, la Klebsiella pneumoniae, en los pacientes internados. ${ }^{7}$ En nuestros pacientes, particularmente en el caso 2, nos enfrentamos a las dificultades diagnósticas de esta entidad. $\mathrm{Al}$ no contar con un recuento de PMN en su ingreso, no pudo realizarse el diagnóstico del EBE. Sin embargo, la presencia de un cultivo monomicrobiano a $E$. coli en la pleurodesis realizada en block quirúrgico (que traduce la representatividad de la muestra), nos hace pensar que este paciente presentó un EBE desde el ingreso previo y con un microorganismo habitual. Los cultivos posteriores a cocáceas positivas los asociamos a una pleura manipulada quirúrgicamente en varias oportunidades.

No existen ensayos clínicos aleatorizados (ECAs) que 
valoren el manejo terapéutico del EBE, por lo que generalmente se extrapolan las medidas terapéuticas de la PBE. ${ }^{1,2}$ El antibiótico debe iniciarse empíricamente luego de realizado el diagnóstico con el recuento de PMN. ${ }^{1}$ Dos elementos definen su elección: el origen del paciente y el perfil de sensibilidad local de los microorganismos. En relación al primer punto, los pacientes pueden tener origen comunitario o relacionado a los cuidados de salud, lo que va a definir el riesgo de aislamiento de microorganismos resistentes a los antimicrobianos, con implicancias pronósticas y terapéuticas. ${ }^{1,3} \mathrm{La}$ prevalencia de microorganismos multirresistentes (MDR) o extremadamente resistentes (XDR) en infecciones en cirróticos aumentó en los últimos años, llegando a un 34\% a nivel mundial según Piano et al., ${ }^{3}$ cifra similar al 37,5\% existente en nuestra región. ${ }^{15}$

En los pacientes con una infección comunitaria y dado que el principal aislamiento está conformado por las enterobacterias, un plan empírico adecuado de primera línea es el de las cefalosporinas de tercera generación (ej. ceftriaxona $2 \mathrm{~g} / \mathrm{día}$ ), pudiendo optar por piperacilina/ tazobactam si existe sospecha de que esté involucrado algún coco positivo como Enterococcus (sensible a la ampicilina). ${ }^{116}$ En el EBE asociado a los cuidados de salud, la piperacilina/tazobactam es la opción si la prevalencia de MDR es baja, o el carbapenem si es alta, siendo en ocasiones necesaria la combinación con glucopéptidos o linezolid si hay alta prevalencia de cocos Gram positivos resistentes a betalactámicos. ${ }^{1}$

$\mathrm{Al}$ igual que en la $\mathrm{PBE}$, es necesario asociar al tratamiento antibiótico la infusión de albúmina $(1,5 \mathrm{~g} / \mathrm{kg}$ de peso al diagnóstico y $1 \mathrm{~g} / \mathrm{kg}$ al día 3) dado que se plantea que disminuye la injuria renal y la mortalidad, aunque no existen estudios al respecto. ${ }^{1,16,17}$

De presentar buena evolución el tratamiento tiene una duración de 5 a 7 días. ${ }^{1}$ No hay certezas si es necesario el control con un nuevo recuento de PMN como en la PBE aunque, como en esta última, sí está indicada la profilaxis secundaria con norfloxacina. ${ }^{1}$

La posibilidad de un tratamiento quirúrgico, como ocurrió en los pacientes presentados, también es un gran desafío, y no exento de complicaciones, siendo un ejemplo el caso $1 .{ }^{16}$ Sin embargo, se debe diferenciar entre el manejo terapéutico del EBE y el manejo del $\mathrm{HH}$ una vez resuelta la complicación infecciosa. En relación al EBE, actualmente no se recomienda la colocación de un tubo de avenamiento pleural ni siquiera en los casos que hay desarrollo bacteriológico en el cultivo del LP. Esto se debe a que se relaciona con un aumento del gasto por el drenaje, la pérdida de proteínas y electrolitos, con la posibilidad de generar complicaciones con disionías e injuria renal, neumotórax o empiema.9, 16, 18 Solo está indicada esta conducta terapéutica de constatarse pus en el espacio pleural. ${ }^{9}, 16,18$

Una vez superada la complicación infecciosa se debe valorar el tratamiento del $\mathrm{HH}$ subyacente, que inicialmente es médico (diuréticos y restricción de $\mathrm{Na}$ ), y toracentesis evacuadora según la necesidad. Cuando la respuesta no es adecuada y la recurrencia del $\mathrm{HH}$ ocurre antes de las 2 semanas, se puede plantear continuar el algoritmo terapéutico. ${ }^{16,19,20}$ En el $\mathrm{HH}$ también la colocación de un tubo de avenamiento pleural se asocia a complicaciones y no es recomendada, llegando a constituir una contraindicación según algunas sociedades científicas internacionales por el incremento de la duración de la estancia hospitalaria y la mortalidad en estos pacientes, siendo en ocasiones preferible el uso del drenaje pleural permanente con un catéter fenestrado, similar al utilizado en derrames pleurales por una patología maligna. ${ }^{19} \mathrm{La}$ primera opción en los pacientes seleccionados es la realización de una derivación portosistémica intrahepática transyugular o TIPS, que presenta una tasa de respuesta favorable en aproximadamente un 70\%. ${ }^{16}, 19,20$ Cuando no existe accesibilidad al TIPS se puede optar por alguna de las opciones quirúrgicas como fue el caso de uno de los pacientes. ${ }^{19}$

Las distintas opciones quirúrgicas son: la pleurodesis química, la reparación de los defectos diafragmáticos (pudiendo asociar una pleurodesis) y la derivación peritoneovenosa o pleuro-venosa. ${ }^{19} \mathrm{La}$ pleurodesis consiste en la ablación del espacio entre la pleura parietal y visceral con una sustancia esclerosante o irritante, como por ejemplo el talco, y se puede realizar mediante una cirugía de tórax videoasistida. ${ }^{16}$ Se la considera en pacientes cuidadosamente seleccionados no candidatos o refractarios al TIPS y puede servir como puente al TH. ${ }^{16,19}$ Las otras opciones, que consisten en la reparación quirúrgica de defectos del diafragma o la derivación quirúrgica, se realizan con menor frecuencia dada la ausencia de experiencia y el riesgo alto de complicaciones comparadas con tratamientos más conservadores. ${ }^{16}$ Es importante destacar el incremento de complicaciones hemorrágicas en estos pacientes, como ocurrió en el caso 1, por lo que se debe ser cauto al optar por dicha terapéutica. ${ }^{16}$

El EBE se asocia a un mal pronóstico de la enfermedad hepática. La mortalidad durante la internación es de un $38,3 \%{ }^{7}$ y posiciona a la cirrosis en al menos un estadio 4 de la clasificación de D' Amico (mortalidad a los 5 años $>50 \%$ ), por lo que es una indicación de evaluación y eventual ingreso a lista para el $\mathrm{TH} .{ }^{21}$ De los dos casos analizados, un paciente falleció, mientras que el otro fue trasplantado. Los elementos de un mal pronóstico en el 
EBE son un MELD elevado al diagnóstico, la internación en cuidados intensivos y la falla del tratamiento antibiótico inicial, ${ }^{7,9}$ de los cuales nuestros pacientes presentaban los dos primeros.

\section{Conclusión}

El EBE es una causa poco frecuente de descompensación de la cirrosis o precipitante de una AoCLF. Existen escasos reportes bibliográficos en nuestro país y la región. Es importante la sospecha diagnóstica en los pacientes con $\mathrm{HH}$ y descompensación de su cirrosis, por lo que es fundamental la solicitud de recuento de PMN y cultivo del LP, dada la ausencia de manifestaciones clínicas, principalmente en la cirrosis avanzada. El manejo terapéutico es complejo, no existen ECAs en relación a las opciones terapéuticas y las mismas se extrapolan de la PBE por las similitudes fisiopatológicas. Se debe ser cauto en la indicación de tratamientos invasivos y la resolución quirúrgica del EBE y el $\mathrm{HH}$ subyacente dado el alto riesgo de complicaciones que estos asocian. La presencia de EBE es una indicación para la evaluación del ingreso a lista de espera para el $\mathrm{TH}$, dada la elevada mortalidad y su mal pronóstico.

Sostén financiero. No se contó con sostén financiero para el estudio.

\section{Aviso de derechos de autor}

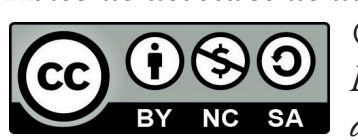

(C) 2021 Acta Gastroenterológica Latinoamericana. Este es un artículo de acceso abierto publicado bajo los términos de la Licencia Creative Commons Attribution (CC BY-NC-SA 4.0), la cual permite el uso, la distribución y la reproducción de forma no comercial, siempre que se cite al autor y la fuente original.

Cite este artículo como: Barceló ME, Prieto Amorín J, Medina Presentado JC y col. Empiema bacteriano espontáneo como complicación de la cirrosis hepática: ¿cuándo sospecharlo y cómo tratarlo? Presentación de casos. Acta Gastroenterol Latinoam. 2021;51(1):100-5. https://doi. org/10.52787/wlhq8266

\section{Referencias}

1. European Association for the Study of the Live. EASL Clinical Practice Guidelines for the management of patients with decompensated cirrhosis. J Hepatol. 2018;69(2):406-60.

2. Runyon BA. Introduction to the revised American Association for the Study of Liver Diseases Practice Guideline management of adult patients with ascites due to cirrhosis 2012. Hepatology. 2013;57(4):1651-3.
3. Piano S, Singh V, Caraceni P, Maiwall R, Alessandria C, Fernandez J, et al. Epidemiology and Effects of Bacterial Infections in Patients With Cirrhosis Worldwide. Gastroenterology. 2019;156(5):1368-80.

4. Castillo C, Benítez C, Baccigaluppi G, Soza A, Riquelme A. Empiema bacteriano espontáneo en paciente cirrótico. Gastr Latinoam. 2004;15(3):203-7.

5. Capriotti A, Rastelli L, Conosciuto JI, Elena S, Tonn F, Basile M, Laborda Molteni J. Pleuritis bacteriana espontánea: complicación de la cirrosis. Rev HPC. 2014;17(1):41-2.

6. Moreau R, Arroyo V. Acute-on-Chronic Liver Failure: A New Clinical Entity. Clin Gastroenterol Hepatol. 2015;13(5):836-41.

7. Chen CH, Shih CM, Chou JW, Liu YH, Hang LW, Hsia TC, et al. Outcome predictors of cirrhotic patients with spontaneous bacterial empyema. Liver Int. 2011;31(3):417-24.

8. Soin S, Sher N, Saleem N. Spontaneous bacterial empyema: An elusive diagnosis in a patient with cirrhosis. BMJ Case Rep. 2018;2018:1-3.

9. Chen TA, Lo GH, Lai KH. Risk factors for spontaneous bacterial empyema in cirrhotic patients with hydrothorax. J Chin Med Assoc. 2003;66(10):579-86.

10. Tu CY, Chen CH. Spontaneous bacterial empyema. Curr Opin Pulm Med. 2012;18(4):355-8.

11. Chertoff J, Nathoo S. Decompensated Liver Cirrhosis Presenting as a Spontaneous Left-Sided Bacterial Empyema. ACG Case Rep J. 2016;3(2):124-6.

12. Xiol X, Castellví JM, Guardiola J, Sesé E, Castellote J, Perelló A, et al. Spontaneous bacterial empyema in cirrhotic patients: A prospective study. Hepatology. 1996;23(4):719-23.

13. Ackerman Z, Reynolds TB. Evaluation of pleural fluid in patients with cirrhosis. J Clin Gastroenterol. 1997;25(4):619-22.

14. Xiol X, Castellote J, Cortes-Beut R, Delgado M, Guardiola J, Sesé E. Usefulness and complications of thoracentesis in cirrhotic patients. Am J Med. 2001;111(1):67-9.

15. Marciano S, Gutierrez Acevedo MN, Palazzo A, Pérez MD, Murga MD, Barbero $S$, et al. Factores asociados a infecciones por organismos multi-resistentes en pacientes con cirrosis: resultados preliminares de un estudio multicéntrico. Congreso Argentino de Hepatología 2019. Buenos Aires, Argentina. Disponible en: http://apps.kingconf.com/hepato2019indice/

16. Lv Y, Han G, Fan D. Hepatic Hydrothorax. Ann Hepatol. 2018;17(1):33-46.

17. Sort P, Navasa M, Arroyo V, Aldeguer X, Planas R, Ruiz-del-Arbol L, et al. Effect of intravenous albumin on renal impairment and mortality in patients with cirrhosis and spontaneous bacterial peritonitis. N Engl J Med. 1999;341(6):403-9.

18. Alonso JC. Pleural effusion in liver disease. Semin Respir Crit Care Med. 2010;31(6):698-705.

19. Banini BA, Alwatari Y, Stovall M, Ogden N, Gershman E, Shah $\mathrm{RD}$, et al. Multidisciplinary Management of Hepatic Hydrothorax in 2020: An Evidence-Based Review and Guidance. Hepatology. 2020;72(5):1851-63.

20. Garbuzenko DV, Arefyev NO. Hepatic hydrothorax: An update and review of the literature. World J Hepatol. 2017; 9(31):1197-204.

21. D’ Amico G, Morabito A, D’Amico M, Pasta L, Malizia G, Rebora P, Valsecchi MG. Clinical states of cirrhosis and competing risks. J Hepatol. 2018;68(3):563-76. 